\title{
Effects of Tolvaptan in patients with acute heart failure: a systematic review and meta- analysis
}

\author{
Chunbin Wang ${ }^{\dagger}$, Bo Xiong ${ }^{\dagger}$ and Lin Cai ${ }^{*}$ (D)
}

\begin{abstract}
Background: Acute heart failure, which requires urgent evaluation and treatment, is a leading cause for admission to the emergency department. The aim of this meta-analysis was to evaluate the effects of tolvaptan on acute heart failure and compare them with the effects of conventional therapy or placebo.

Methods: The electronic databases PubMed, EMBASE, and the Cochrane Controlled Trial registry were searched from their starting dates to October 24, 2016. Two authors independently read the trials and extracted related information from the included studies. We used fixed-effects or random-effects models to assess the overall combined risk estimates according to $\mathrm{I}^{2}$ statistics. Analysis to determine sensitivity and publication bias was conducted.
\end{abstract}

Results: Six randomised controlled trials from eight articles, with a total of 746 patients, were included for analysis. Compared with the control, tolvaptan reduced body weight in two days (WMD 1.35; $95 \% \mathrm{Cl} 0.75$ to 1.96), elevated sodium level in two days (WMD 2.33; 95\% Cl 1.08 to 3.57) and five days (WMD 1.57; $95 \% \mathrm{Cl} 0$. 04 to 3.09), and ameliorated symptoms of dyspnoea (RR 0.82; $95 \% \mathrm{Cl} 0.71-0.95$ ). However, tolvaptan did not improve long-term (RR 1.04; $95 \% \mathrm{Cl} 0.66-1.62)$ or short-term all-cause mortality (RR $0.89 ; 95 \% \mathrm{Cl} 0.45-1.76$ ), incidence of clinical events (worsening heart failure, RR $0.75 ; 95 \% \mathrm{Cl} 0.50-1.12$ and worsening renal function, RR 0.97; 95\% Cl 0.75-1.27), and length of hospital stay in patients (WMD 0.14; 95\% Cl -0.29 to 2.38) with acute heart failure.

Conclusion: Tolvaptan can decrease body weight, increase serum sodium level, and ameliorate some of the congestion symptoms in patients with acute heart failure, which may help avoid the overdose of loop diuretics, especially in patients with renal dysfunction.

Keywords: Heart failure, Acute, Tolvaptan, Therapy, Meta-analysis

\section{Background}

Heart failure (HF), characterised by volume overload in both extravascular and intravascular spaces, is a common clinical syndrome. It is one of leading causes of admission to the emergency departments, distressing approximately $1-2 \%$ of adult population in developed countries [1]. Acute heart failure (AHF) refers to the exacerbation of $\mathrm{HF}$, requiring urgent hospital admission for evaluation and treatment. Although advances in

\footnotetext{
* Correspondence: cd3yycailin@163.com

${ }^{\dagger}$ Equal contributors

Department of Cardiology, The Third People's Hospital of Chengdu,The

Second Affiliated Chengdu Clinical College of Chongqing Medical University, Chengdu 610031, Sichuan, China
}

medicine and devices for AHF treatment have reduced AHF-related mortality, hospitalisation rates remain high. There are nearly six million HF patients in America, contributing to one million emergency department visits and over one million hospitalisations annually $[2,3]$. Congestion is the major reason for hospitalisation of patients with AHF [4], and severe congestion means poor . Even with the use of diuretics and vasodilators, congestion persists in many AHF patients, and it 6]. Loop diuretics is the first-line therapy for AHF despite serious adverse effects such as decrease in renal function and activation of the sympathetic nervous 
system and renin-angiotensin-aldosterone system [7]. Additionally, loop diuretics are known to be associated with hyponatremia, renal dysfunction, and hypotension due to loss of intravascular volume owing to sodium depletion [8].

Tolvaptan (TLV), an oral vasopressin $\mathrm{V}_{2}$-receptor antagonist, results in the clearance of free water. Previous studies have shown that TLV is a safe and effective drug for the treatment of euvolemic and hypervolemic hyponatremia as well as for patients with $\operatorname{HF}[9,10]$. Three meta-analyses were performed to investigate the efficacy and safety of TLV treatment in patients hospitalised for HF [11-13]; however, these analyses were primarily focused on chronic heart failure (CHF). Since the publication of these meta-analyses, many new clinical trials have been performed to investigate the efficacy and safety of TVL in patients with AHF $[8,14,15]$. To assess the effects of TLV on AHF completely, we conducted a meta-analysis of randomised controlled trials (RCTs) focusing on the effects of TLV in patients with AHF in comparison with the effects of conventional therapy or placebo.

\section{Methods}

This meta-analysis was conducted in compliance with the Preferred Reporting Items for Systematic Reviews and Meta-Analysis (PRISMA) statement [16].

\section{Search strategy}

We searched the databases of PubMed, EMBASE, and the Cochrane Controlled Trial registry from their starting dates to October 24, 2016 using the following keywords: acute decompensated heart failure OR acute heart failure AND tolvaptan OR vasopressin $\mathrm{V}_{2}$-receptor blocker. Our article search was restricted to studies involving human subjects and those published in English. The full search strategies for PubMed are provided in Additional file 1.

\section{Study selection}

We first excluded the reduplicated studies using Endnote software, and then screened the studies according to the titles or abstracts. Two authors (Wang Chunbin and Xiong Bo) scanned the titles and abstracts of all retrieved articles independently, and irrelevant studies were excluded at this stage. The eligibility of the remaining articles was further evaluated for disagreement or uncertainty. Disagreements were resolved by discussion or consensus of a third reviewer.

\section{Inclusion criteria}

The inclusion criteria for the studies were as follows: it should (1) be a randomised, controlled trial (RCT); (2) include participants who are adult patients with AHF, defined as patients had dyspnea at rest requiring urgent hospital admission for evaluation and treatment;(3) compare TLV with controls or other diuretic agents; and (4) include any relevant outcomes: all-cause mortality, clinical events, sodium level, dyspnoea improvement, body weight reduction, and fluid loss.

\section{Exclusion criteria}

The exclusion criteria were as follows: (1) observational study and (2) study on CHF or not reporting the desired outcome.

\section{Data extraction}

Data extraction was performed independently by two authors (Wang Chunbin and Xiong Bo), and the data were checked by a third reviewer. Any disagreements were settled by discussion.

The following information was extracted from each retrieved article: characteristics of included studies (title, first author, publication year, journal, corresponding address, study design, inclusion and exclusion criteria, dose of TLV, treatment duration, and pertinent outcomes).

\section{Assessment of risk of bias}

Risk of bias for included studies was independently assessed by two reviewers by the Cochrane risk of bias tool [17]. Disagreements were resolved by discussion.

\section{Statistical analysis}

We used Stata 12.0 (Stata Statistical Software: Release 12. StataCorp LP, College Station, TX) and Revman software (version 5.3, Cochrane Collaboration, Oxford, United Kingdom) for analyses. Heterogeneity was evaluated by $\mathrm{I}^{2}$ test $\left(\mathrm{I}^{2}>50 \%\right.$ indicating significant heterogeneity). If there was no significant heterogeneity among the included studies, inverse variance (IV) fixed-effect model was utilised; otherwise, random-effects model was used. In addition, sensitivity analysis was performed to identify the stability of statistical data, and publication bias was evaluated by funnel plots. $P<0.05$ was considered statistically significant.

\section{Results}

Six-hundred ninety-one articles were identified from the database research: 226 of PubMed, 417 of EMBASE, and 48 of the Cochrane Library. After screening the titles and abstracts, 28 studies eligible for full text screening were identified. A full-text evaluation was performed and 20 were excluded for the following reasons: data published as reviews $(n=2)$, meta-analyses $(n=3)$, study performed on CHF patients $(n=5)$, and failure to report required endpoints $(n=10)$. Finally, six RCTs among eight 
articles were included. The flow diagram of study selection is shown in Fig. 1.

\section{Study characteristics and quality}

The study characteristics of the six RCTs in the USA, South America, Europe, and Japan from 2012 to 2016, recruiting 746 patients, are presented in Table 1 . The mean follow-up duration of the studies was 2 to 636 days. The dose of TLV ranged from 3.75 to $30 \mathrm{mg} /$ day. Two of the studies used placebo as control treatment, three used carperitide or furosemide, and one study compared TLV treatment with conventional therapy. Most participants had AHF [Left ventricular ejection fraction (LVEF) < 50\%] of New York Heart Association (NYHA) class II-IV. The risk of bias was evaluated with the Cochrane risk of bias tool. Most items for all included RCTs showed low risk; however, there was insufficient information in some studies, which made the evaluation difficult. Overall, the RCTs included in our meta-analysis were of relatively high quality, except one study by Matsue et al, which showed a high risk of bias [14]. The results are summarised in Fig. 2.
Effect of TLV on all-cause mortality and length of hospital stay

Six studies reported all-cause mortality of AHF after TLV therapy, two reported short-term ( $\leq 30$ days) and three reported long-term ( $>30$ days) all-cause mortality. Compared with the control, TLV had no impact on long-term (RR 1.04; 95\% CI 0.66-1.62) or short-term allcause mortality (RR 0.89 ; 95\% CI $0.45-1.76$ ) in patients with AHF (Fig. 3).

Three studies reported the effect of TLV on the length of hospital stay. The meta-analysis indicated that TLV treatment had no effect on the length of hospital stay (WMD 0.14; 95\% CI -0.29 to 2.38) (Fig. 4).

\section{Effect of TLV on clinical events}

Six RCTs reported the effect of TLV on clinical events, three on worsening heart failure (WHF), and four on worsening renal function (WRF). Compared with the control treatments, TLV was not likely to reduce the clinical events of WHF (RR 0.75; 95\% CI 0.50-1.12) (Fig. 5) or WRF (RR 0.97; 95\% CI 0.751.27; Fig. 6).

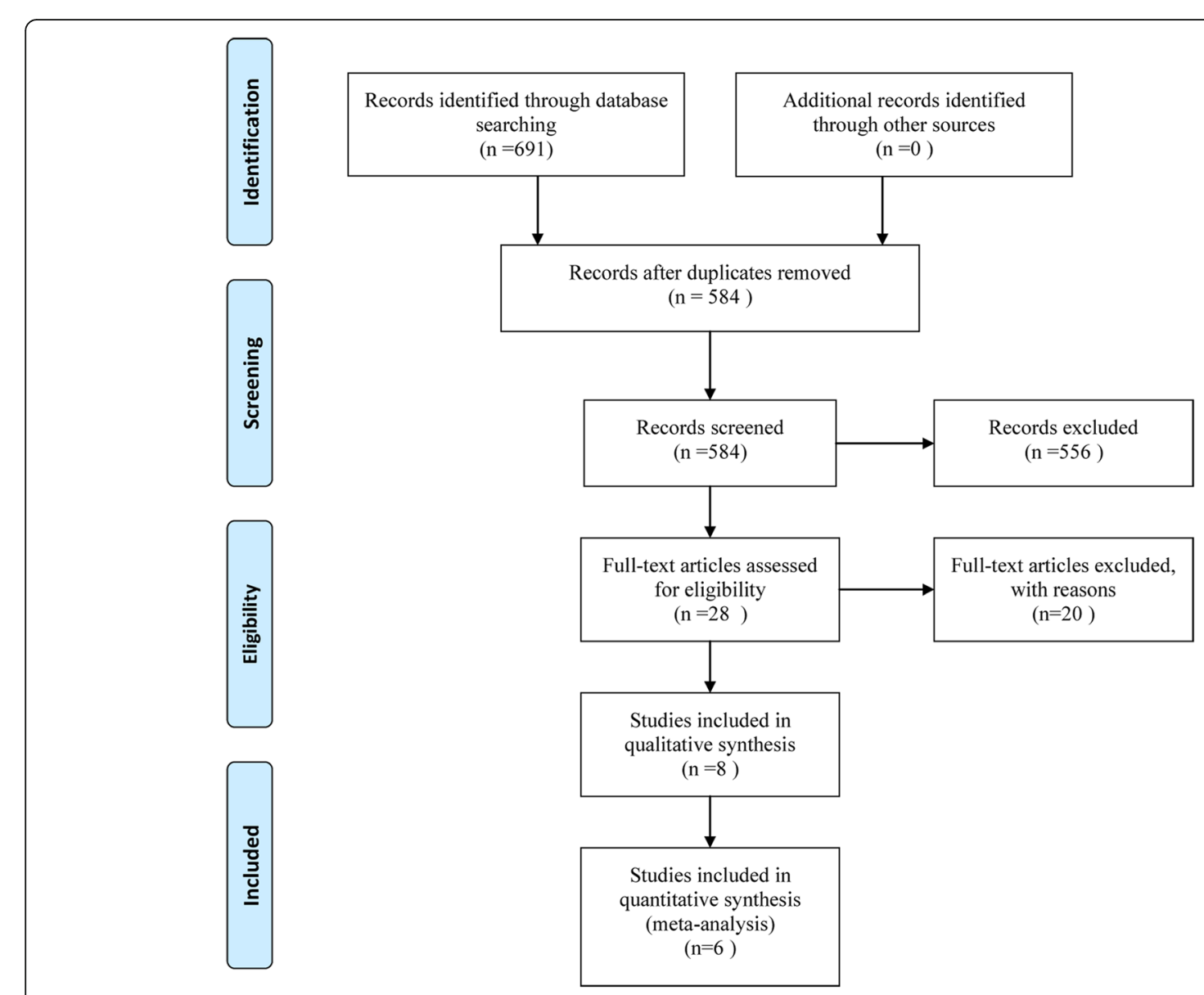

Fig. 1 PRISMA flow diagram of study selection 


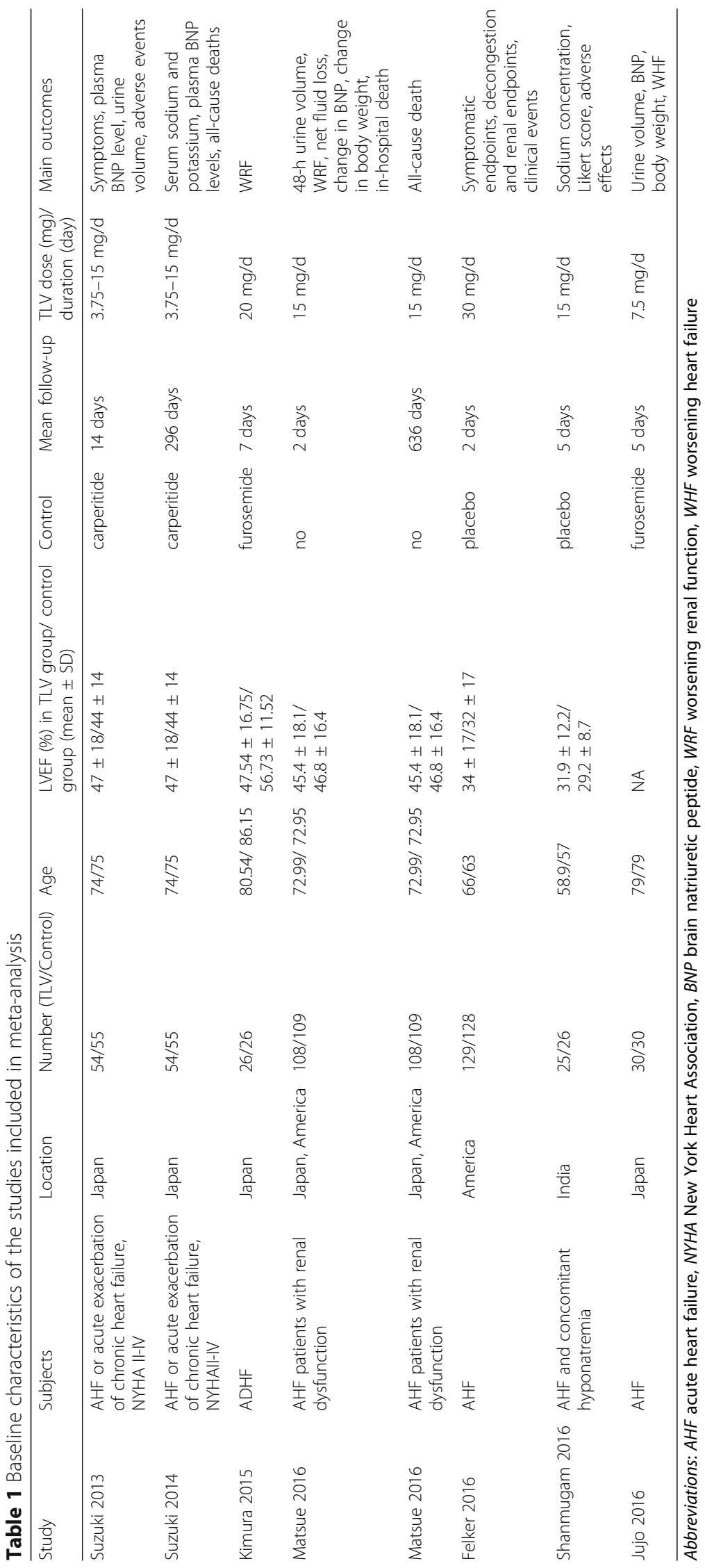




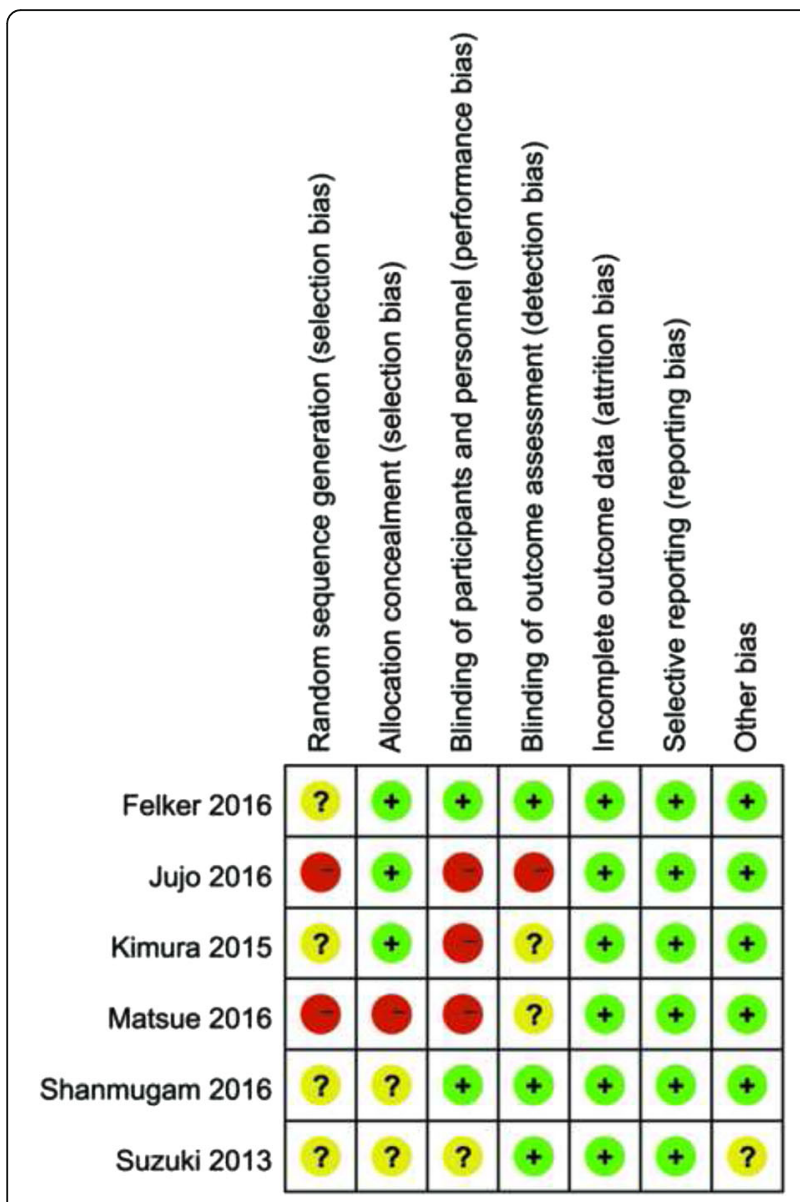

Fig. 2 Risk of bias summary
In subgroup analysis, the control in two study (Kimura 2015, Jujo 2016) were furosemide, in other two study were placebo (Felker 2016,Shanmugam 2016) and no drug in one study (Matsue 2016). In the placebo group, TLV had no effect on WRF (RR 1.35, 95\% CL 0.931.96), but in the furosemide group, TLV decreased the rate of WRF (RR 0.45,0.23-0.89) (Fig. 6).

\section{Effect of TLV on dyspnoea improvement}

Only two studies demonstrated the effect of TLV on dyspnoea at $6,8,12,24$, and $48 \mathrm{~h}$. The pooled result showed statistical significance (RR $0.82 ; 95 \%$ CI $0.71-$ 0.95) (Fig. 7).

\section{Effect of TLV on mean body weight reductions and fluid loss}

Mean body weight and fluid loss reflected the aquaretic effect of TLV in AHF patients. In our analysis, TLV could significantly lower the mean body weight two days (WMD 1.35; 95\% CI 0.75 to 1.96; Fig. 8). The analysis of fluid loss in two days also showed statistical significance (WMD 0.66; 95\% CI 0.35 to 0.98, Fig. 8).

\section{Effect of TLV on sodium level}

Three studies reported the effect of TLV on sodium level at one, two, three, and five days. The results showed statistical significance at two days (WMD 2.33; 95\% CI 1.08 to 3.57 ; Fig. 9) and five days (WMD 1.57; $95 \% \mathrm{CI}$ 0.04 to 3.09; Fig. 9).

Publication bias was not observed for all outcomes in the funnel plots for the analysis of mortality (Fig.10) and clinical events (Fig. 11). Sensitive analyses were performed to investigate the influence of a single study on the overall risk estimate and test the stability of the study. The results did not show substantial difference.

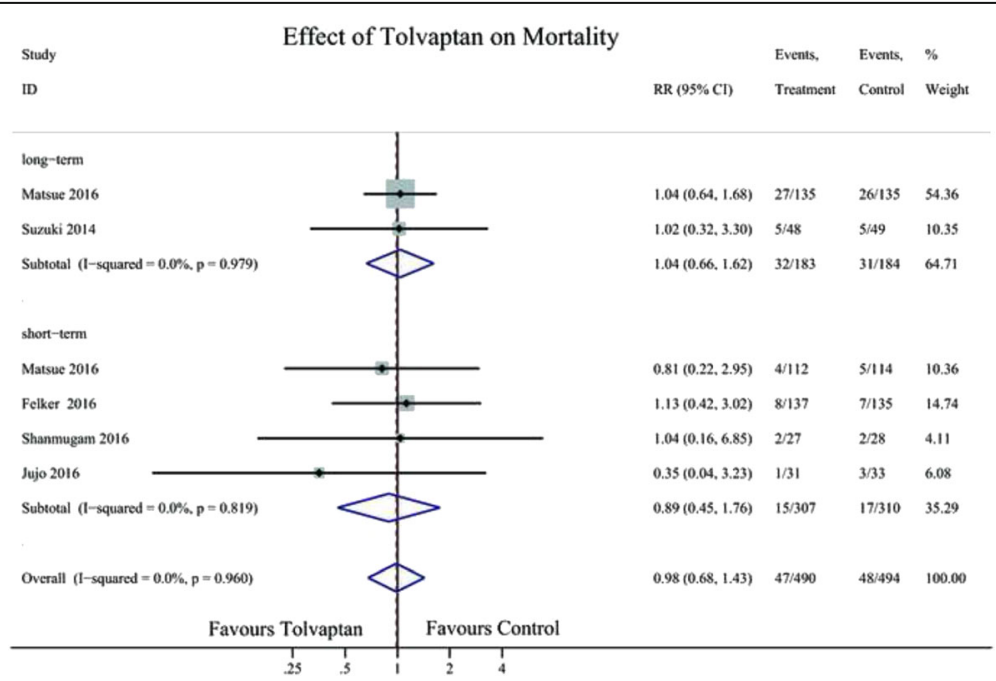

Fig. 3 Forest plot depicting the effect of tolvaptan on mortality and clinical events versus control. RR, rate ratio; Cl, confidence interval 


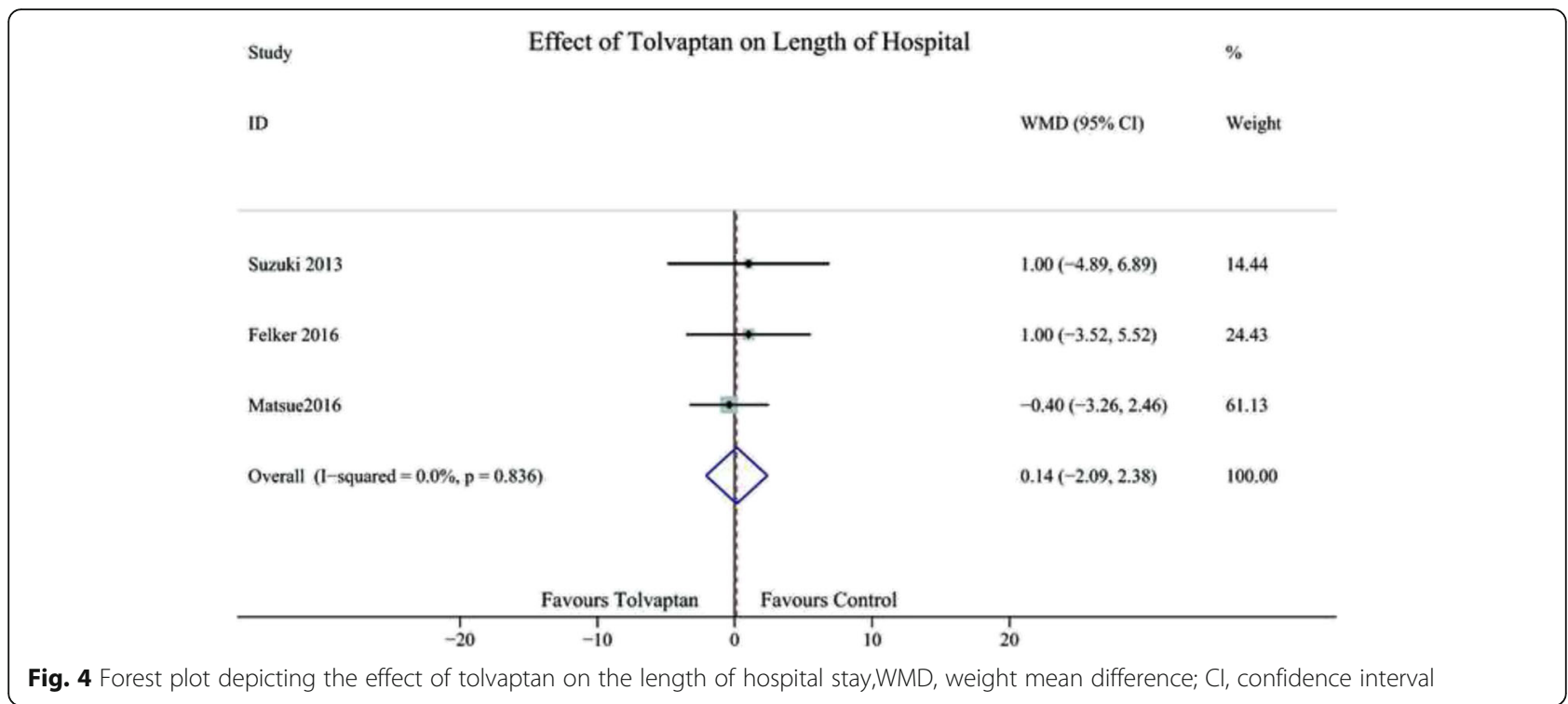

\section{Discussion}

The main findings of this meta-analysis indicate that TLV could reduce body weight, elevate sodium level, and ameliorate symptoms of dyspnoea in patients with AHF. However, TLV did not ameliorate or worsen all-cause mortality, incidence of clinical events (WHF, WRF), and length of hospital stay in patients with AHF.

Congestion is the main reason for hospitalisation of patients with AHF, and decongestion is essential for treating patients with AHF. Loop diuretics are routinely used for natriuresis,timely administration of intravenous loop diuretics is able to save the patient's life by efficaciously reducing the clinical congestion and immediately decreasing the hemodynamic overload. But high dose of loop diuretics related to renal function deterioration and long-term adverse clinical outcomes [18-20].
TLV, which induces aquaresis, is also effective for decongestion. A retrospective study of 102 consecutive patients with decompensated HF, who were treated with TLV, showed that the early use of TLV reduced the length of hospital stay and mortality [21]. However, in our analysis, all-cause mortality and length of hospital stay had no statistical significance; the mean body weight decreased and sodium concentration increased. To date, only chronic therapy with neurohormonal antagonists, such as $\beta$-blockers, angiotensin converting enzyme inhibitors, and angiotensin II receptor blockades, improved clinical outcomes in patients with HF. The results of our meta-analysis showed that TLV as an adjunctive therapy in clinical setting might improve the acute symptoms of dyspnoea; however, it may have no effect on the length of hospital stay or post-discharge

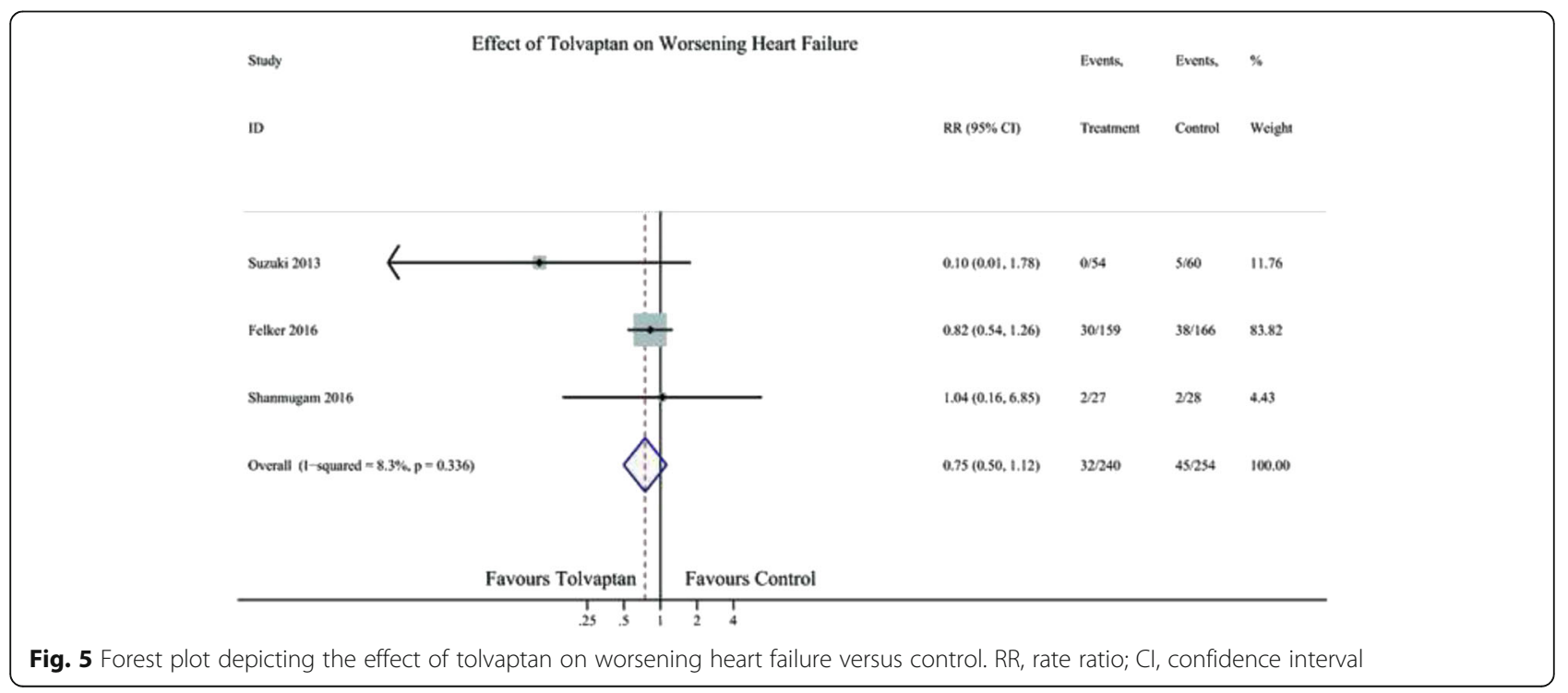




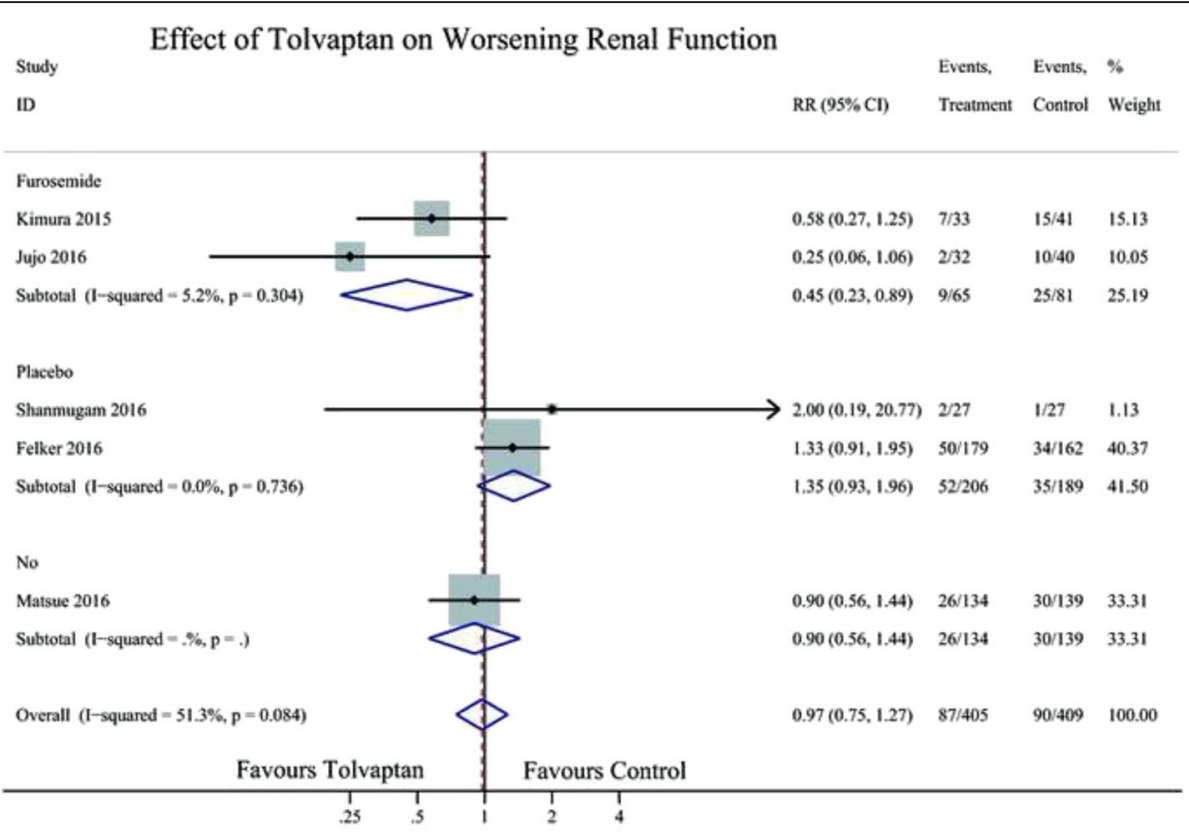

Fig. 6 Forest plot depicting the effect of tolvaptan on worsening renal function versus control. RR, rate ratio; Cl, confidence interval

outcomes. Hence, the development of better strategies for the treatment of AHF remains a challenge.

Our meta-analysis suggested that TLV could elevate the serum sodium level in patients with AHF in two days. Studies have also shown that decreased sodium is a critical predictor of survival in patients with HF [22]. Being a non-peptide vasopressin type-2 receptor antagonist, TLV is now available for patients with HF having hyponatremia. The study by Shanmugam et al. [8] revealed that TLV at a dose of $15 \mathrm{mg} /$ day is effective in reversing hyponatremia, when administered over a period of five days, indicating that TLV was more suitable for AHF with hyponatremia.
In our analysis, TLV had no effect on the clinical events, WHF and WRF. WHF was defined as worsening of signs or symptoms of HF necessitating an increase in HF treatment. In-hospital WHF could lead to a poor prognosis for AHF patients-both in terms of rehospitalisation and mortality [23, 24]. In the TACTICS-HF study, 90\% WHF was treated with additional loop diuretics, and it has been related to a near doubling of post-discharge event rates [23]. Therefore, the use of TLV in AHF may avoid the need for additional loop diuretics and reduce post-discharge event rates.

Renal dysfunction is also a common comorbidity in AHF patients, and it forebodes higher rates of mortality

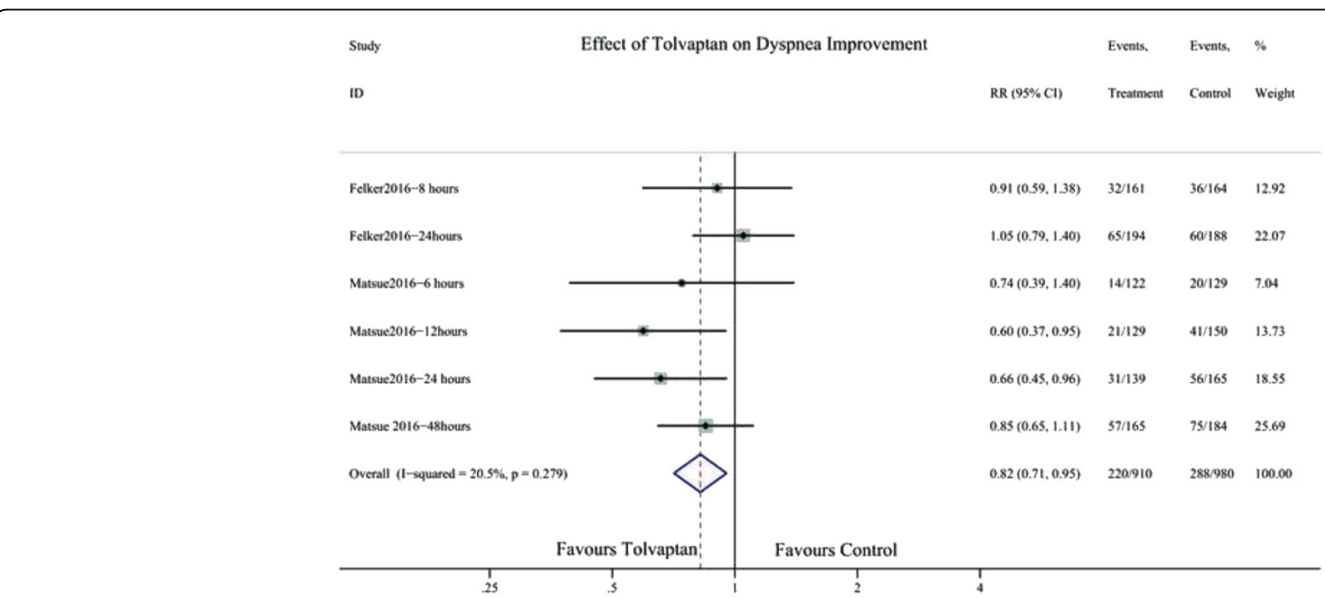

Fig. 7 Forest plot depicting the effect of tolvaptan on dyspnoea improvement versus control. RR, rate ratio; Cl, confidence interval 


\begin{tabular}{|c|c|c|c|}
\hline \multicolumn{3}{|c|}{ Effect of TLV on Body Weight Reductions and Fluid Loss } & \multirow{2}{*}{$\begin{array}{l}\% \\
\text { Weight }\end{array}$} \\
\hline ID & & WMD $(95 \% \mathrm{Cl})$ & \\
\hline \multicolumn{4}{|l|}{ Body weight reductions } \\
\hline Felker 2016-2day & & $2.60(0.92,4.28)$ & 12.89 \\
\hline Matsue2016-2days & $\longrightarrow$ & $1.17(0.52,1.82)$ & 87.11 \\
\hline Subtotal $(\mathrm{I}-\mathrm{squared}=58.8 \%, \mathrm{p}=0.119)$ & & $1.35(0.75,1.96)$ & 100.00 \\
\hline \multicolumn{4}{|l|}{ Fluid loss } \\
\hline Felker 2016-2days & $\rightarrow$ & $0.53(0.16,0.90)$ & 72.98 \\
\hline Matsue2016-2days & $\longrightarrow$ & $1.02(0.41,1.63)$ & 27.02 \\
\hline Subtotal $(\mathrm{I}-\mathrm{squared}=45.6 \%, \mathrm{p}=0.175)$ & & $0.66(0.35,0.98)$ & 100.00 \\
\hline Favours Control & Favours Tolvaptan & & \\
\hline
\end{tabular}

Fig. 8 Forest plot depicting the effect of tolvaptan on body weight reductions and fluid loss versus control. WMD, weight mean difference; $\mathrm{Cl}$, confidence interval

and hospitalisation in patients with AHF to a great extent [25]. A previous meta-analysis showed that TLV elevated the serum creatinine level slightly [11]; however, there was no significant difference in morbidity associated with renal dysfunction between TLV and control groups in EVEREST study [26]. The changes in creatinine levels that occur during successful decongestion therapy do not necessarily indicate the same adverse prognosis [27]. However, renal impairment and WRF, defined as an increase in serum creatinine of $0.3 \mathrm{mg} / \mathrm{dL}$ from baseline within 7 days from admission, are often induced by the overuse of loop diuretics [28]. They are associated with increased morbidity and mortality. Renal protective treatment could greatly improve the prognosis of HF patients [29]. In a previous small retrospective study, addition of TLV resulted in more urine output

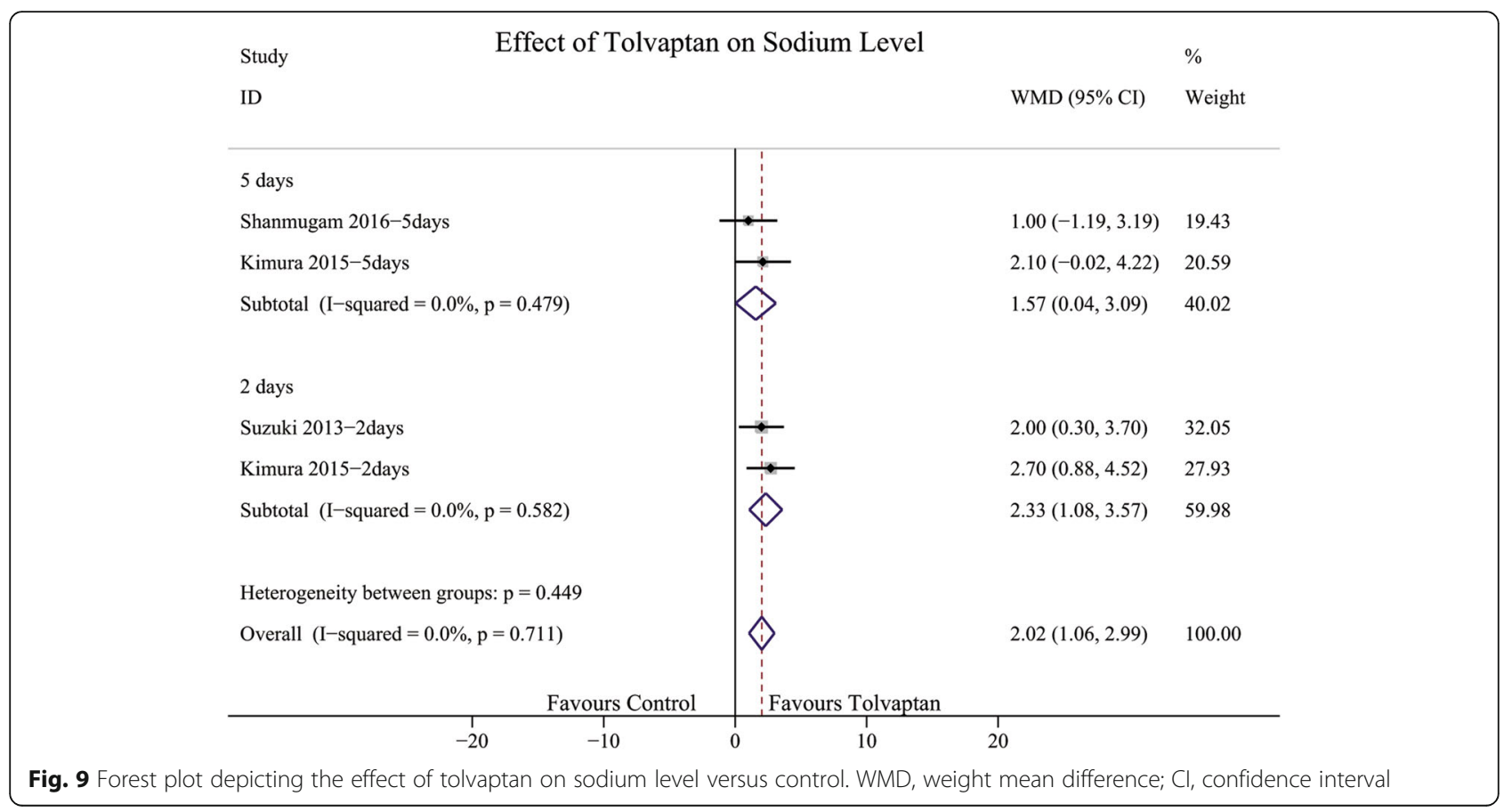




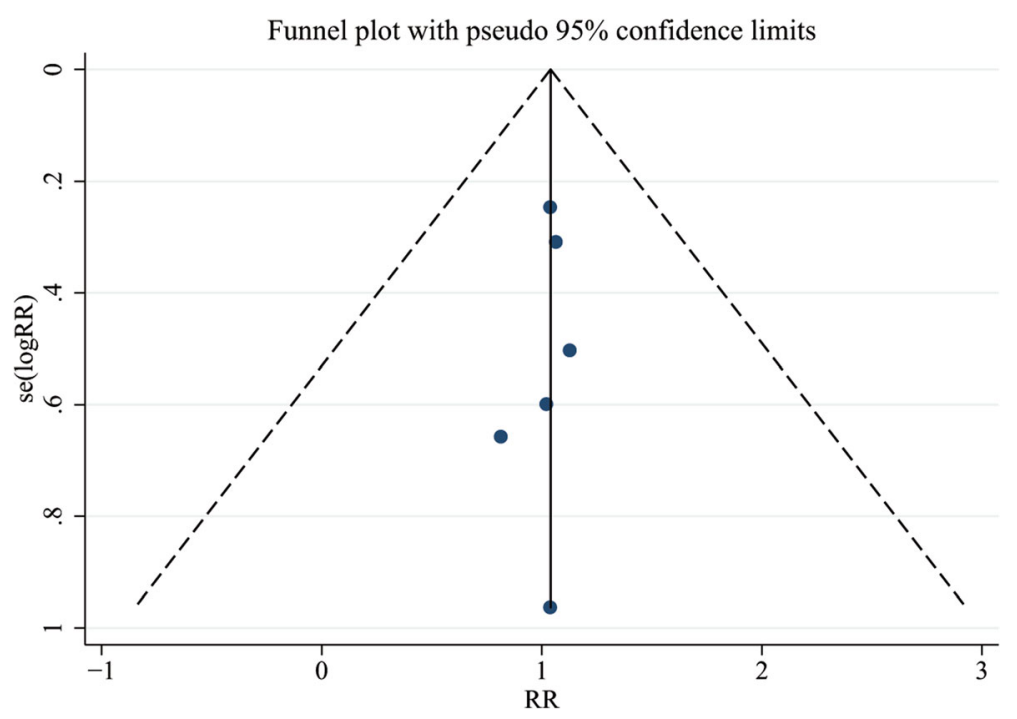

Fig. 10 Funnel plot for analysis of all-cause mortality with pseudo 95\% confidence limits

and less WRF compared with that of furosemide [30]. The AQUAMARINE study showed that treatment with TLV resulted in more urine output and dyspnoea relief compared to that with conventional therapy; however, there was no significant difference in the rate of WRF between the groups [14]. In our analysis, although TLV had no effect on WRF overall, while in the subgroup of furosemide, TLV decreased the rate of WRF. The results indicated that use of TLV in AHF might reduce WRF compared with the administration of loop diuretics.

\section{Limitations}

Potential limitations of our meta-analysis should be considered. First, limited number of RCTs was included in our meta-analysis. Only two studies from the selected trials measured long-term mortality and four studies had the outcome of short-term mortality. Second, the duration of TLV use and follow-up time were different in each included study, and this might affect the clinical outcomes. Third, differences in race, age, and complication among studies may result in slightly diverse response to therapy. Fourth, different control treatments might also lead to inaccurate results.

\section{Conclusions}

We observed that TLV did not reduce all-cause mortality, length of hospital stay, and clinical events of AHF. However, the use of TLV could decrease body weight,

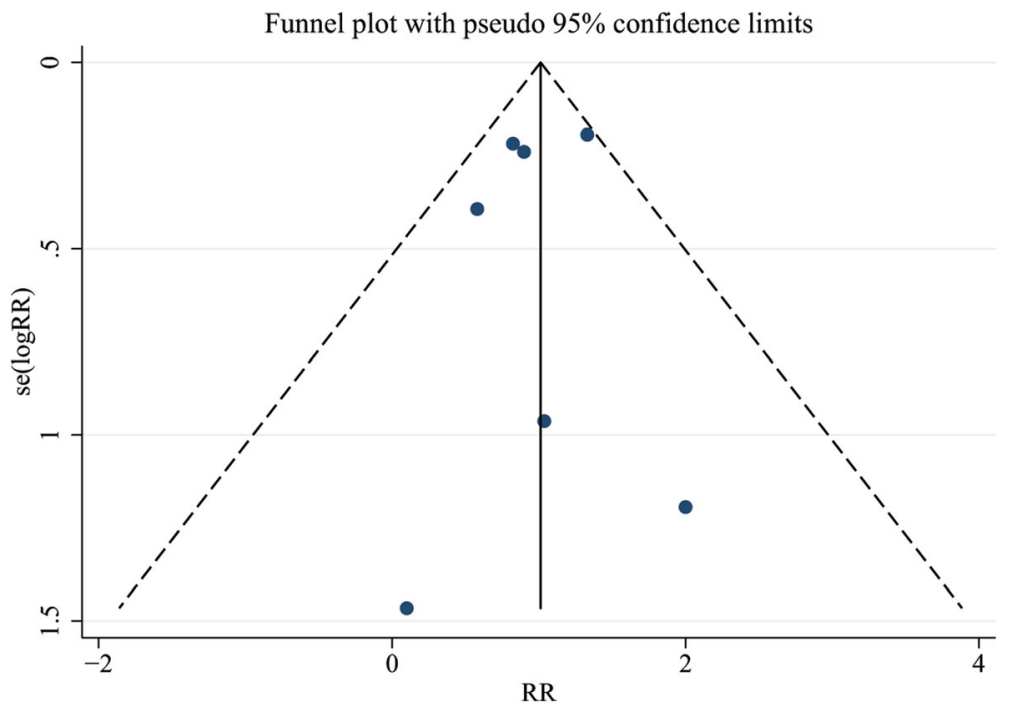

Fig. 11 Funnel plot for analysis of clinical events with pseudo 95\% confidence limits 
increase serum sodium level, and ameliorate some of the congestion symptoms, which may avoid the disadvantages of loop diuretics, especially in patients with renal dysfunction. Overall, TLV could be a selective treatment for AHF.

\section{Additional file}

Additional file 1: Search Strategy in PubMed. (DOCX 15 kb)

\section{Abbreviations}

ADHF: Acute decompensated heart failure; AHF: Acute heart failure; BNP: Btype natriuretic peptide; CHF: Chronic heart failure; LVEF: Left ventricular ejection fraction; NA: Not available; NYHA: New York Heart Association; RCT: Randomisedcontrolled trial; RR: Relative risk; TVL: Tolvaptan; WHF: Worsening heart failure; WMD: Weighted mean difference; WRF: Worsening renal function

\section{Acknowledgments}

None.

\section{Funding}

The authors received no funding for this work.

\section{Availability of data and materials}

Data and materials presented in the main paper or additional supporting files.

\section{Authors' contributions}

$\mathrm{CL}$ drafted the manuscript. WC and XB analysed the data, performed statistical analysis, and wrote the paper. All authors contributed to the development of the selection criteria, the risk of bias assessment strategy, data extraction criteria, search strategy, and statistical expertise. All the authors read, provided feedback, and approved the final manuscript.

\section{Competing interests}

All authors declare that they have no conflict of interest.

\section{Consent for publication}

Not applicable.

Ethics approval and consent to participate

Ethical approval was not applicable for this systematic review and meta- analysis.

\section{Publisher's Note}

Springer Nature remains neutral with regard to jurisdictional claims in published maps and institutional affiliations.

Received: 14 December 2016 Accepted: 14 June 2017

Published online: 20 June 2017

\section{References}

1. Authors/Task Force M, Document R: 2016 ESC Guidelines for the diagnosis and treatment of acute and chronic heart failure: The Task Force for the diagnosis and treatment of acute and chronic heart failure of the European Society of Cardiology (ESC). Developed with the special contribution of the Heart Failure Association (HFA) of the ESC. Eur J Heart Fail. 2016;18(8):891975 .

2. Roger VL, Go AS, Lloyd-Jones DM, Adams RJ, Berry JD, Brown TM, et al. Heart disease and stroke statistics-2011 update: a report from the American Heart Association. Circulation. 2011;123(4):e18-e209.

3. Storrow $A B$, Jenkins $C A$, Self $W H$, Alexander PT, Barrett TW, Han JH, et al. The burden of acute heart failure on U.S. emergency departments. JACC Heart failure. 2014;2(3):269-77.

4. Gheorghiade M, Follath F, Ponikowski P, Barsuk JH, Blair JE, Cleland JG, et al. Assessing and grading congestion in acute heart failure: a scientific statement from the acute heart failure committee of the heart failure association of the European Society of Cardiology and endorsed by the
European Society of Intensive Care Medicine. Eur J Heart Fail. 2010;12(5): 423-33.

5. Mullens W, Abrahams Z, Francis GS, Sokos G, Taylor DO, Starling RC, et al. Importance of venous congestion for worsening of renal function in advanced decompensated heart failure. J Am Coll Cardiol. 2009;53(7):589-96.

6. Ambrosy AP, Pang PS, Khan S, Konstam MA, Fonarow GC, Traver B, et al. Clinical course and predictive value of congestion during hospitalization in patients admitted for worsening signs and symptoms of heart failure with reduced ejection fraction: findings from the EVEREST trial. Eur Heart J. 2013;34(11):835-43.

7. Jujo K, Saito K, Ishida I, Furuki Y, Kim A, Suzuki Y, et al. Randomized pilot tria comparing tolvaptan with furosemide on renal and neurohumoral effects in acute heart failure. ESC heart failure. 2016:3(3):177-88.

8. Shanmugam E, Doss CRMP, George M, Jena A, Rajaram M, Ramaraj B, et al. Effect of tolvaptan on acute heart failure with hyponatremia - a randomized double blind, controlled clinical trial. Indian Heart J. 2016;68:S15-21.

9. Konstam MA, Gheorghiade M, Burnett JC Jr, Grinfeld L, Maggioni AP, Swedberg K, et al. Effects of oral tolvaptan in patients hospitalized for worsening heart failure: the EVEREST outcome trial. JAMA. 2007:297(12): 1319-31.

10. Udelson JE, Orlandi C, Ouyang J, Krasa H, Zimmer CA, Frivold G, et al. Acute hemodynamic effects of tolvaptan, a vasopressin V2 receptor blocker, in patients with symptomatic heart failure and systolic dysfunction: an international, multicenter, randomized, placebo-controlled trial. J Am Coll Cardiol. 2008;52:1540-5.

11. Xiong B, Huang Y, Tan J, Yao Y, Wang C, Qian J, et al. The short-term and long-term effects of tolvaptan in patients with heart failure: a meta-analysis of randomized controlled trials. Heart Fail Rev. 2015;20(6):633-42.

12. Yang CJ, Yang J, Yang J, Fan ZX. Arginine vasopressin antagonist tolvaptan in the treatment of heart failure: a meta-analysis of randomized controlled trials. Int J Clin Exp Med. 2015;8(12):22117-28.

13. Alskaf $\mathrm{E}$, Tridente $\mathrm{A}$, Al-Mohammad A. Tolvaptan for heart failure, systematic review and meta-analysis of trials. J Cardiovasc Pharmacol. 2016;68(3):196-203.

14. Matsue Y, Suzuki M, Torii S, Yamaguchi S, Fukamizu S, Ono Y, et al. Clinical effectiveness of Tolvaptan in patients with acute heart failure and renal dysfunction. J Card Fail. 2016;22(6):423-32.

15. Felker GM, Mentz RJ, Cole R, Adams KF, Egnaczyk GF, Fiuzat M, et al. Efficacy and safety of Tolvaptan in patients hospitalized with acute heart failure. $J$ Am Coll Cardiol. 2016;69(11):1399-406.

16. Liberati A, Altman DG, Tetzlaff J, Mulrow C, Gotzsche PC, loannidis JP, et al. The PRISMA statement for reporting systematic reviews and meta-analyses of studies that evaluate health care interventions: explanation and elaboration. Ann Intern Med. 2009:151(4):W65-94.

17. Higgins JP, Altman DG, Gotzsche PC, Juni P, Moher D, Oxman AD, et al. The Cochrane Collaboration's tool for assessing risk of bias in randomised trials. BMJ. 2011;343:d5928.

18. Domanski M, Norman J, Pitt B, Haigney M, Hanlon S, Peyster E. Diuretic use, progressive heart failure, and death in patients in the studies of Left ventricular dysfunction (SOLVD). J Am Coll Cardiol. 2003:42(4):705-8.

19. Ahmed A, Husain A, Love TE, Gambassi G, Dell'Italia LJ, Francis GS, et al. Heart failure, chronic diuretic use, and increase in mortality and hospitalization: an observational study using propensity score methods. Eur Heart J. 2006:27(12):1431-9.

20. Kapelios CJ, Kaldara E, Ntalianis A, Sousonis V, Repasos E, Sfakianaki T, Vakrou S, Pantsios C, Nanas JN, Terrovitis JV High furosemide dose has detrimental effects on survival of patients with stable heart failure 2015, 56(2):154-159.

21. Matsukawa R, Kubota T, Okabe M, Yamamoto Y. Early use of V2 receptor antagonists is associated with a shorter hospital stay and reduction in inhospital death in patients with decompensated heart failure. Heart Vessel. 2016;31(10):1650-8.

22. Felker GM, Leimberger JD, Califf RM, Cuffe MS, Massie BM, Adams KF Jr, et al. Risk stratification after hospitalization for decompensated heart failure. $J$ Card Fail. 2004;10(6):460-6.

23. Kelly JP, Mentz RJ, Hasselblad V, Ezekowitz JA, Armstrong PW, Zannad F, et al. Worsening heart failure during hospitalization for acute heart failure: insights from the acute study of clinical effectiveness of Nesiritide in decompensated heart failure (ASCEND-HF). Am Heart J. 2015;170(2):298-305.

24. Davison BA, Metra M, Cotter G, Massie BM, Cleland JG, Dittrich HC, et al. Worsening heart failure following admission for acute heart failure: a pooled analysis of the PROTECT and RELAX-AHF studies. JACC Heart failure. 2015;3(5):395-403 
25. Damman K, Navis G, Voors AA, Asselbergs FW, Smilde TD, Cleland JG, et al. Worsening renal function and prognosis in heart failure: systematic review and meta-analysis. J Card Fail. 2007;13(8):599-608.

26. Gheorghiade M, Konstam MA, Burnett JC Jr, Grinfeld L, Maggioni AP, Swedberg K, et al. Short-term clinical effects of tolvaptan, an oral vasopressin antagonist, in patients hospitalized for heart failure: the EVEREST clinical status trials. JAMA. 2007;297(12):1332-43.

27. Metra M, Davison B, Bettari L, Sun H, Edwards C, Lazzarini V, et al. Is worsening renal function an ominous prognostic sign in patients with acute heart failure? The role of congestion and its interaction with renal function. Circulation Heart failure. 2012;5(1):54-62.

28. Metra M, Nodari S, Parrinello G, Bordonali T, Bugatti S, Danesi R, et al. Worsening renal function in patients hospitalised for acute heart failure: clinical implications and prognostic significance. Eur J Heart Fail. 2008:10(2):188-95.

29. Damman K, Valente MA, Voors AA, O'Connor CM, van Veldhuisen DJ, Hillege $\mathrm{HL}$. Renal impairment, worsening renal function, and outcome in patients with heart failure: an updated meta-analysis. Eur Heart J. 2014;35(7):455-69.

30. Matsue $Y$, Suzuki M, Seya M, Iwatsuka R, Mizukami A, Nagahori W, et al. Tolvaptan reduces the risk of worsening renal function in patients with acute decompensated heart failure in high-risk population. J Cardiol. 2013;61(2):169-74

Submit your next manuscript to BioMed Central and we will help you at every step:

- We accept pre-submission inquiries

- Our selector tool helps you to find the most relevant journal

- We provide round the clock customer support

- Convenient online submission

- Thorough peer review

- Inclusion in PubMed and all major indexing services

- Maximum visibility for your research

Submit your manuscript at www.biomedcentral.com/submit
Biomed Central 